\title{
El portafolio como recurso de aprendi- zaje e instrumento de evaluación de estudiantes repetidores de enfermería
}

\author{
The portfolio as a learning resource and evaluation tool for course repeating \\ students of the nursing school
}

Manuel L. Vera Cortés y $\mathrm{M}^{\mathrm{a}}$ del Coro Canalejas Pérez

Escuela Universitaria de Enfermería La Paz. Universidad Autónoma. Madrid

El objetivo de la experiencia educativa descrita en este artículo, es la utilización del portafolios como recurso para impulsar la autodirección de los estudiantes en el proceso de aprender a aprender y potenciar la construcción personal del conocimiento. Material y métodos: La experiencia se desarrolló durante tres cursos académicos en la Escuela Universitaria de Enfermería La Paz (Universidad Autónoma. Madrid). El portafolios fue elaborado por 21 estudiantes matriculados en la asignatura Ecología Humana y Salud Comunitaria, que no la habían superado en el curso anterior. Se llevó a cabo en dos fases, una de diseño y otra de aplicación de este recurso.

Resultados: A excepción de un alumno, todos progresaron, con diferentes grados de excelencia, en la construcción del conocimiento y en la capacidad de autodirigir su aprendizaje, destacando la utilidad del portafolios para comprender los conceptos fundamentales de la asignatura y adquirir competencias como aprendices autodirigidos.

Conclusiones: Se han logrado los objetivos planteados al iniciar la experiencia y consideramos conveniente realizarla con estudiantes repetidores ya que la evaluación formativa y el aprendizaje a través del diálogo con el profesor y sus compañeros les facilita la progresiva construcción de nuevos conocimientos e incrementa sus destrezas metacognitivas.

Para utilizar el portafolios con un número elevado de alumnos, sería necesario que lo elaboraran en pequeños grupos y que se implicaran varios tutores para el seguimiento del proceso de aprendizaje de los estudiantes.

Palabras clave: Portafolios, aprender a aprender, aprendizaje constructivo, aprendizaje autodirigido, aprendizaje activo, autoevaluación, evaluación formativa.

The goal of this educative experience is to use the portfolio as a tool to drive self-direction in the learning to learn process and to stimulate individual knowledge construction.

Material and methods: The experience was undertaken throughout three academic years at the Nursing School 
of La Paz (Universidad Autónoma. Madrid). The portfolio was made for twenty one students enrolled in the Human Ecology and Community Health class. None of them was having obtained a pass grade during last year course. The experience was developed in two phases, the design phase and the tool application phase.

Results: All students, excepting one, made progress with different excellence degrees in critical knowledge elaboration and developing the capability to drive self-direction of learning process. All of them emphasized the usefulness of the experience to understand the most important guidelines of the course and to acquire competences as auto-guided learners. Conclusions: The objectives set for the portfolio experience have been achieved, and we believe it will be convenient to carry it out with rehearsing students. The formative assessment and the dialogue whit teacher or between students facilitate a progressively build capabilities to understand new concepts and increase metacognitive skills development. Using the portfolio with a higher number of students would require working it out in small groups and that several tutors took care of the learning process of the students.

Key words: Portfolio, learning to learn, constructive learning, self directed learning, active learning, self assessment, formative assessment

\section{INTRODUCCIÓN}

Un portafolios es un instrumento que contribuye a potenciar la autodirección del estudiante, impulsar la construcción del conocimiento y enfatizar la individualización del proceso de aprendizaje-enseñanza, constituyendo una alternativa de evaluación frente al examen convencional ${ }^{1}$. Está compuesto por una recopilación significativa y organizada de trabajos del estudiante que evidencian su progreso, esfuerzo y logros. Por tanto, permite conocer su forma de razonar, recoge información del producto y fundamentalmente del proceso de aprendizaje e involucra al alumno en la evaluación ${ }^{2}$.

Por esta razón, se decidió utilizarlo con estudiantes repetidores de la asignatura troncal de primer curso de la Diplomatura de Enfermería "Ecología Humana y Salud Comunitaria". Estos se caracterizaban por tener dificultades para fundamentar, analizar, sintetizar y relacionar conceptos; disponer de tiempo muy limitado para llevar a cabo las actividades presenciales de la asignatura y ser poco activos en su aprendizaje, con un modelo de enseñanza-aprendizaje centrado en la transmisión del conocimiento por parte del profesor.

$\mathrm{Al}$ igual que otros autores ${ }^{3,4}$, con la utilización de este recurso se pretendía llevar a cabo una experiencia enmarcada en el paradigma reflexivo, con el fin de valorar su eficacia para:

Impulsar la autodirección de los estudiantes en el proceso de aprender a aprender.

Potenciar la construcción personal del conocimiento.

Contribuir al desarrollo de la capacidad de análisis-síntesis, la creatividad y el pensamiento crítico.

Involucrar al estudiante en el proceso de evaluación.

\section{MATERIAL Y MÉTODOS}

\section{Sujetos de estudio}

Esta experiencia se desarrolló durante los cursos académicos 2003-2004, 2004-2005 y 2005-2006 en la Escuela Universitaria de Enfermería La Paz, en la asignatura Ecología Humana y Salud Comunitaria. Se propuso como opción a los estudiantes que no habían superado los objetivos de la asignatura en el curso anterior, por presentar dificultades de aprendizaje y de disponibilidad de tiempo en las actividades presenciales desarrolladas:

Curso 2003-2004: 12 estudiantes.

Curso 2004-2005: 11 estudiantes.

Curso 2005-2006: 6 estudiantes.

De los 29 estudiantes a los que se ofertó la experiencia, participaron 21. Los 8 que decidieron no participar, por inseguridad para realizarlo y falta de tiempo, llevaron a cabo las actividades planificadas para el resto de alumnos matriculados en la asignatura.

Se seleccionó el portafolio por considerar que era un instrumento útil para que este grupo realizara un aprendizaje significativo de las competencias específicas y genéricas, a cuyo logro contribuye esta materia, sin necesidad de asistir a las actividades presenciales planificadas en cada curso para los 80 alumnos restantes. Con estos últimos no se utilizó dicha metodología por la imposibilidad de que un solo profesor llevara a cabo, en un número tan ele- 
vado de estudiantes, el seguimiento continuo del proceso de aprendizaje que requiere el portafolio.

\section{Metodología}

Durante los tres cursos, la experiencia se llevó a cabo en dos fases:

\section{FASE DE DISEÑO}

En esta fase se realizó el diseño del portafolio, decidiendo el tipo más adecuado para integrar aprendizaje, enseñanza y evaluación de manera continua. Se llevó a cabo en los meses previos al inicio del curso, planificando:

Componentes del portafolios: Siguiendo a Font Ribas $^{5}$ se decidió que el portafolio incluyera evidencias de aprendizaje obligatorias y opcionales, con el fin de que aquellos estudiantes más motivados pudieran reflejar su esfuerzo personal. Las evidencias obligatorias eran:

Evidencias de contenido: elaboración de contenidos relacionando conceptos e identificando sus implicaciones en el proceso de cuidar, búsqueda y selección bibliográfica y realización de casos prácticos.

Evidencias de proceso: Análisis de objetivos logrados el curso anterior, planteamiento de nuevos objetivos, autoevaluación continua y gestión del tiempo de aprendizaje.

Períodos de elaboración del portafolio y calendario de tutorías: Se planificaron tres entregas a lo largo del curso, coincidiendo con el final de cada trimestre.

Teniendo en cuenta que la discusión y reflexión con el profesor y los compañeros facilita el proceso de aprendizaje, se optó por la realización de tutorías en grupo e individuales, entendidas como una "conversación de aprendizaje" que involucra a los estudiantes en un diálogo evaluativo muy eficaz.

Contenido de la "Guía del portafolio": Fue diferente para los distintos periodos de elaboración, en general, estaba compuesta por: fines, criterios para la elaboración y evaluación, calendario de tutorías, objetivos y esquema de contenidos por tema, casos prácticos a trabajar en grupo, cuadro orientador para la planificación del tiempo a dedicar a las diferentes actividades de aprendizaje, bibliografía básica y lecturas de apoyo.

Criterios de evaluación del portafolio: Para evaluar los portafolios en las tres revisiones llevadas a cabo a lo largo del curso, se diseñó un documento-guía ${ }^{7}$ (Tabla 1) que reflejaba las dimensiones y características a analizar en función de los objetivos pretendidos con la utilización de dicho instrumento y se establecieron los niveles de excelencia (Tabla 2).. A
Tabla 1. Criterios para la evaluación del portafolios.

CONSTRUCCIÓN DEL CONOCIMIENTO (capacidad de análisis, síntesis, creatividad y pensamiento crítico)

Elaboración personal de ideas y conceptos. Comprensión del contenido de la materia. Interrelación de conceptos.

Búsqueda de conexión teoría-práctica. Selección de ideas principales y secundarias. Grado de profundización en los conceptos tratados. Fundamentación teórica de argumentos. Originalidad y profundidad de las reflexiones.

\section{PRESENTACIÓN}

Estructura y organización del portafolios.

Expresión escrita y utilización de un lenguaje profesional.

\section{AUTODIRECCIÓN DEL APRENDIZAJE}

Análisis de las dificultades de aprendizaje.

Planteamiento de objetivos de aprendizaje a partir de las dificultades señaladas.

Evaluación del logro de los objetivos planteados.

Planificación y gestión del tiempo de aprendizaje.

Cantidad y pertinencia de la bibliografía aportada.

Aportación de evidencias diferentes a las propuestas por el profesor.

Para que los estudiantes evaluaran la experiencia, se diseñó un cuestionario cerrado de autoevaluación del grado de adquisición de las competencias y dos preguntas abiertas sobre ventajas e inconvenientes de esta forma de aprender.

\section{FASE DE CONSTRUCCIÓN Y DESARROLLO.}

$\mathrm{Al}$ inicio se realizaron dos sesiones para explicar en qué consistía la experiencia y entregarles la guía de elaboración. Los estudiantes realizaron los portafolios individualmente, de Octubre a Junio, en tres períodos que coincidieron con los tres trimestres del curso. Los contenidos a trabajar en cada periodo se centraban:

Primer periodo: La persona y su entorno.

Concepción de salud.

Segundo periodo: Condicionantes del nivel

de salud.

Tercer periodo: El cuidado de la salud de

la comunidad.

Como actividades de apoyo se realizaron en cada trimestre "tutorías planificadas", que fueron disminuyendo a lo largo del curso con el fin de potenciar la autodirección del estudiante, y tutorías "a demanda”, presenciales o por correo electrónico, para la orientación y evaluación formativa. Al final de cada 


\section{Tabla 2. Niveles de excelencia en la elaboración del portafolios}

\section{NIVEL 1: Insuficiente}

CONSTRUCCIÓN DEL CONOCIMIENTO(Capacidad de análisis, síntesis, pensamiento crítico, creatividad).Los documentos elaborados demuestran poca comprensión de la materia y son una copia de los textos utilizados. No hay interrelación de conocimientos. No se diferencian ideas principales y secundarias. Las afirmaciones reflexivas no están fundamentadas con argumentos.

PRESENTACIÓN: No se cumplen los requisitos. Hay frecuentes errores en la expresión escrita. No está bien organizado.

AUTODIRECCIÓN DEL APRENDIZAJE. No se hace un análisis de dificultades, no se plantean objetivos de mejora, no se gestiona adecuadamente el tiempo de aprendizaje. Se limitan a cumplir la tarea propuesta por el profesor y no hacen búsquedas bibliográficas o aportan algunas referencias diferentes a las propuestas por el profesor que no son pertinentes.

\section{NIVEL 2: Suficiente}

CONSTRUCCIÓN DEL CONOCIMIENTO (Capacidad de análisis, síntesis, pensamiento crítico, creatividad): Los documentos elaborados demuestran una adecuada asimilación del contenido. Se interrelacionan algunos conceptos. Se diferencian ideas principales y secundarias. Hay poca evidencia de reflexión independiente, con un mínimo intento de análisis y síntesis. PRESENTACIÓN: Organización incompleta y lenguaje profesional mejorable.

AUTODIRECCIÓN DEL APRENDIZAJE. Se hace un análisis de dificultades, no se plantean objetivos de mejora, no se gestiona adecuadamente el tiempo de aprendizaje. Aportan algunas referencias diferentes a las propuestas por el profesor que son pertinentes, aunque no las utilizan mucho en la fundamentación.

\section{NIVEL 3: BIEN}

CONSTRUCCIÓN DEL CONOCIMIENTO (Capacidad de análisis, síntesis, pensamiento crítico; creatividad): Los documentos elaborados demuestran una sólida comprensión del contenido. Hay interrelación de conocimientos, aunque no analizan las implicaciones en la práctica del cuidado. Se diferencian ideas principales y secundarias. Los puntos de vista del estudiante se apoyan en argumentos y referencias adecuados. PRESENTACIÓN: Bien estructurado, documentado correctamente y con un lenguaje fluido y profesional.

AUTODIRECCIÓN DEL APRENDIZAJE. Se hace un análisis de dificultades, se plantean algunos objetivos de mejora, se gestiona adecuadamente el tiempo de aprendizaje. Aportan algunas referencias diferentes a las propuestas por el profesor que son muy pertinentes.

\section{NIVEL 4 Muy bien}

CONSTRUCCIÓN DEL CONOCIMIENTO (Capacidad de análisis, síntesis, pensamiento crítico, creatividad). Los documentos elaborados muestran muy buena compresión del contenido de la materia. Hay interrelación de conocimientos. Se diferencian ideas principales y secundarias. Las reflexiones demuestran un razonamiento original y en profundidad. Los argumentos utilizados están sustentados por referencias bien fundamentadas. PRESENTACIÓN: Bien estructurado. Se expresa de forma muy correcta en el lenguaje profesional.

AUTODIRECCIÓN DEL APRENDIZAJE. Se hace un análisis de dificultades, se plantean objetivos de mejora y los evalúan, se gestiona adecuadamente el tiempo de aprendizaje. Aportan evidencias diferentes a las propuestas por el profesor y seleccionan bastantes referencias bibliográficas nuevas muy pertinentes.

Tabla 3. Nivel de excelencia obtenido por los alumnos en cada entrega

\begin{tabular}{|c|l|cccc|}
\hline \multicolumn{2}{|c|}{} & \multicolumn{3}{c|}{ Número de alumnos por nivel de excelencia } \\
\hline Entregas & Criterios & Insuficiente & Suficiente & Bien & Muy bien \\
\hline Primera & Construcción & 13 & 8 & 0 & 0 \\
& Presentación & 17 & 4 & 0 & 0 \\
& Autodirección & 11 & 7 & 3 & 0 \\
\hline Segunda & Construcción & 6 & 13 & 2 & 0 \\
& Presentación & 8 & 10 & 3 & 0 \\
& Autodirección & 5 & 11 & 5 & 0 \\
\hline \multirow{2}{*}{ Tercera } & Construcción & 1 & 11 & 9 & 0 \\
& Presentación & 1 & 3 & 17 & 0 \\
& Autodirección & 1 & 10 & 10 & 0 \\
\hline
\end{tabular}

trimestre se realizaba una tutoría individual con el fin de realizar una coevaluación con el estudiante y analizar los logros conseguidos y los aspectos a mejorar en siguientes períodos.

A lo largo de cada curso se realizaron 5 tutorías planificadas en grupo, antes de la primera entrega, 4 antes de la segunda y 3 antes de la tercera. Así mismo se llevaron a cabo un promedio de 15 tutorías a demanda.

\section{RESULTADOS}

\section{Evaluación de cada entrega}

Con la elaboración del portafolio, a excepción de 1 estudiante, los 20 restantes progresaron en la adquisición de competencias y superaron la asignatura con diferente grado de excelencia (Tabla 3). Del análisis de los portafolios en las diferentes entregas, destacan los siguientes datos de evolución:

CONSTRUCCIÓN DEL CONOCIMIENTO: Al inicio de la experiencia todos elaboraron documen- 
tos limitándose a copiar los textos utilizados, sin relacionar conceptos ni establecer conexiones teóricas y prácticas, mostrando poca evidencia de reflexión independiente. Al final 20 estudiantes habían progresado en estos aspectos, mostrando 11 todavía dificultades para interrelacionar los conceptos con el cuidado enfermero y relacionar teoría y práctica.

PRESENTACIÓN: En la primera entrega 17 estudiantes mostraron dificultades para organizar el contenido del portafolio y utilizaban un lenguaje cotidiano. Al finalizar 20 habían progresado, destacando especialmente 17 por la estructura final y la utilización del lenguaje profesional.

AUTODIRECCIÓN DEL APRENDIZAJE: Al comienzo 11 estudiantes mostraron un alto grado de dependencia del profesor, de manera que no se plantearon objetivos de mejora y gestionaron mal el tiempo y los recursos de aprendizaje. Al finalizar la experiencia, 10 habían progresado bastante y otros 10, aunque habían progresado tenían todavía dificultad para utilizar recursos diferentes a los aportados por el profesor y evaluar el logro de objetivos.

Todos los estudiantes tuvieron dificultades para seleccionar evidencias opcionales, de manera que sólo 8 aportaron algunas: lecturas comentadas, selección de fotografías, elaboración de gráficos, asistencia a talleres específicos, valoración del domicilio y de la situación de salud del grupo familiar.

\section{Opinión de los estudiantes}

Al finalizar el curso, los 21 estudiantes realizaron la evaluación de la experiencia. De la autoevaluación del grado de adquisición de competencias, según una escala Likert de 5 puntos (1-muy poco-, 2 -insuficiente-, 3-suficiente-, 4-bastante-, 5-mucho-), destaca:

Construcción del conocimiento (Figura 1): Las competencias que consideraron que habían conseguido en mayor grado fueron "comprender los conceptos fundamentales" (valor medio: 4,1), "identificar las ideas y conceptos nucleares" (valor medio: 4,3) e "identificar la importancia de la asignatura para la formación de enfermeras" (valor medio: 4). Las competencias adquiridas en menor grado fueron "desarrollo del pensamiento crítico" y "capacidad para interrelacionar conceptos", obteniendo ambas un valor medio de 3,5.

Autodirección del aprendizaje: (Figura 2): Las competencias que consideraron habían adquirido en mayor grado fueron "aprender de forma autónoma", "establecer una relación con el profesor como facili-

\section{Figura 1. Resultados de la autoevaluación de competencias adquiridas con la elaboración del portafolios (Valor medio en una escala Likert de 1 a 5)}

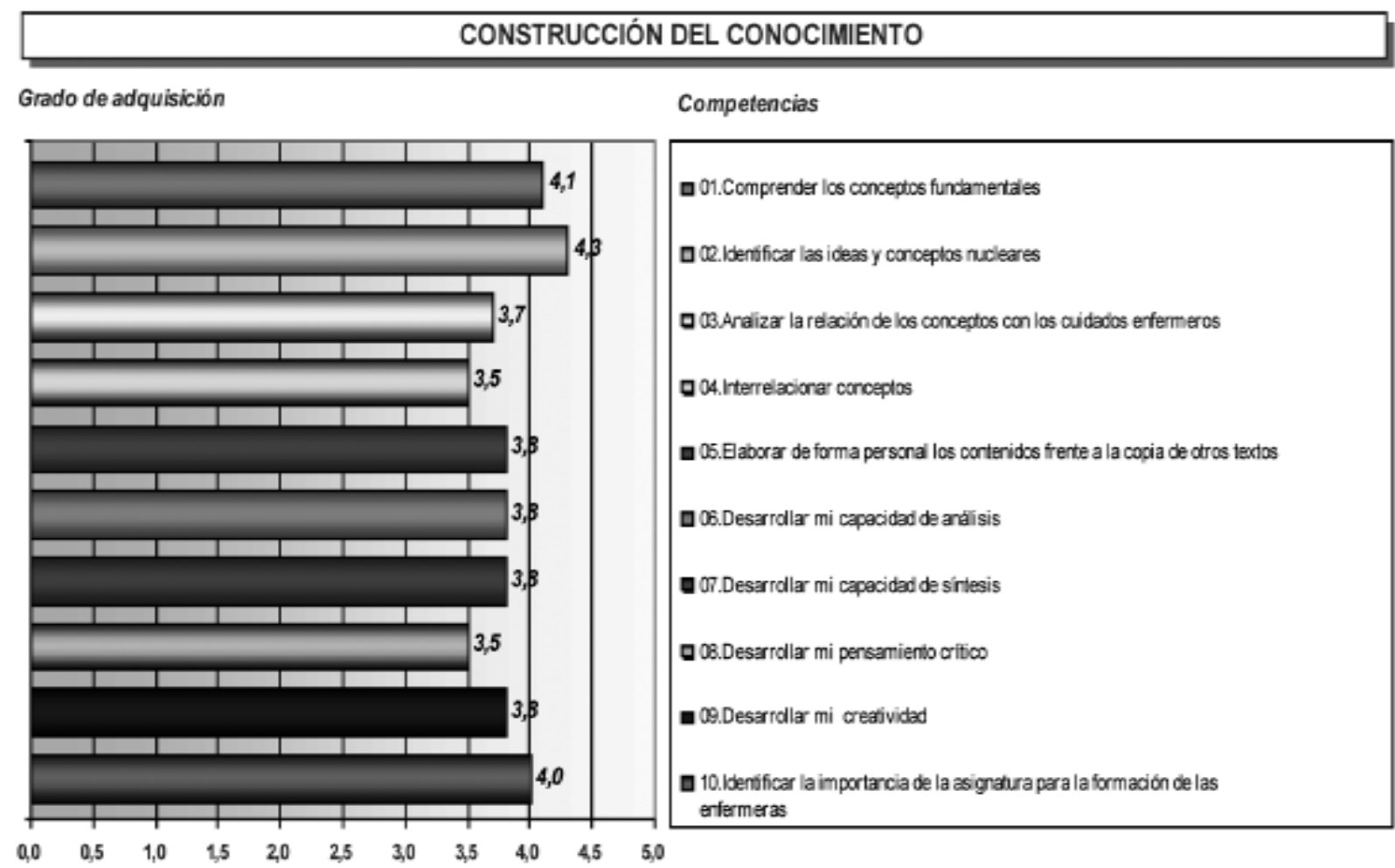


Figura 2. Resultados de la autoevaluación de competencias adquiridas con la elaboración del portafolios (Valor medio en una escala Likert de 1 a 5 ).

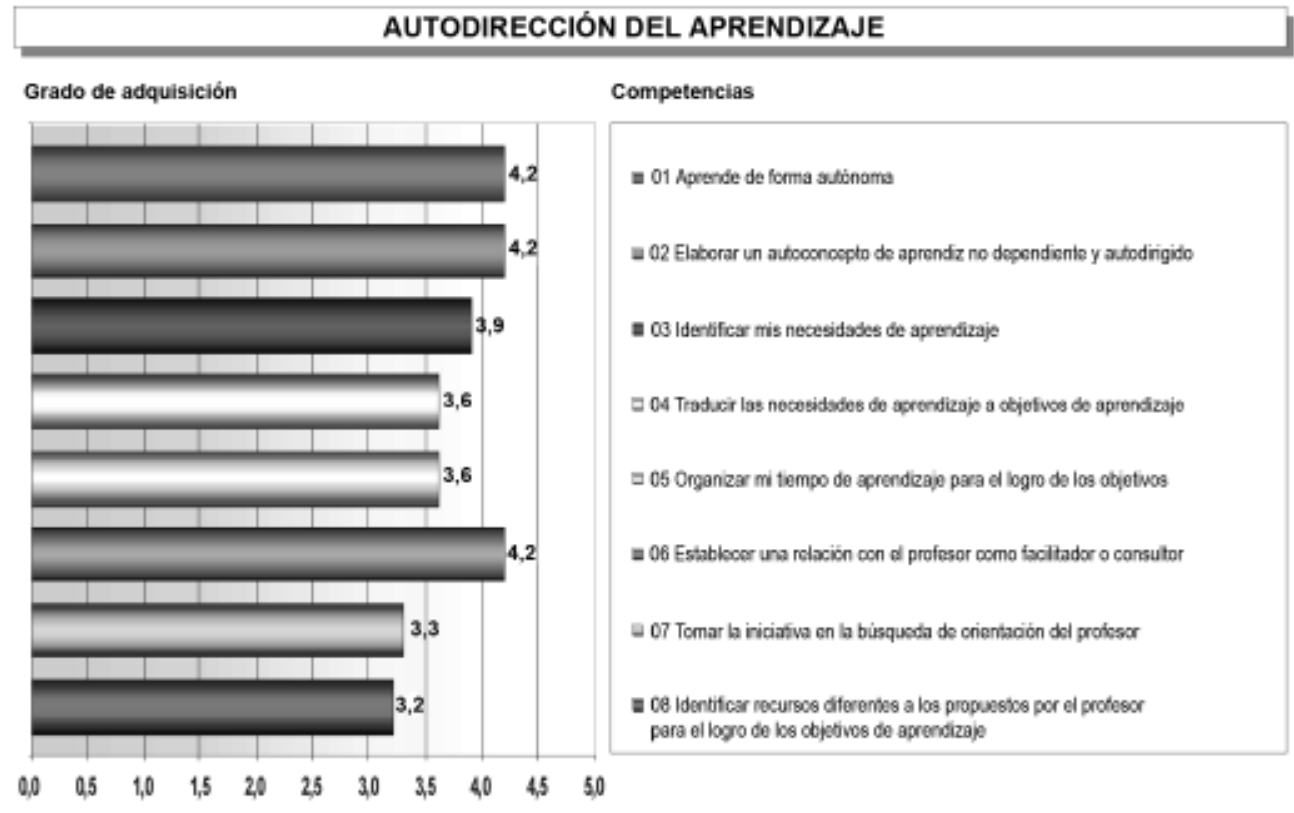

tador o consultor" y "elaborar un autoconcepto como aprendiz no dependiente y autodirigido", obteniendo todas un valor medio de 4,2. Adquirieron en menor grado capacidad para "tomar la iniciativa en la búsqueda de orientación del profesor" (valor medio: 3,3) e "identificar recursos diferentes a los propuestos" (valor medio: 3,2 )

En las preguntas abiertas los estudiantes destacaron como principales ventajas de la utilización del portafolio:

Facilita la comprensión de los conceptos fundamentales y su relación entre sí.

Las tutorías sirven de guía para identificar los puntos fundamentales del trabajo, orientar la reflexión y la crítica fundamentada y corregir errores.

Mejora la capacidad de organización y gestión del tiempo.

Contribuye a desarrollar la capacidad para trabajar en grupo.

Mejora la capacidad de búsqueda, análisis y selección bibliográfica

Permite aprender a aprender

Asimismo destacaron las siguientes dificultades:

Requiere mucho tiempo para su elaboración.

La búsqueda y selección bibliográfica produce inseguridad.

Al inicio del trabajo, fue difícil el diseño del portafolio y la elaboración de conceptos.

Manejo informático en la elaboración del trabajo.
Establecer relaciones conceptuales

Búsqueda de implicaciones enfermeras

Genera inseguridad inicial.

Mayor esfuerzo tanto nuestro como del profesor

\section{Horas de dedicación de estudiantes y profesor}

El tiempo utilizado por los estudiantes para la elaboración del portafolio estuvo comprendido en un intervalo de 150 a 200 horas, con excepción de 2 que emplearon un promedio de 400 horas.

Por parte del profesor, se emplearon entre 70-90 horas, repartidas entre el diseño, tutorías y evaluación de portafolios.

\section{DISCUSIÓN}

Basándonos en esta experiencia, coincidimos con otros autores ${ }^{8,9}$ en que la utilización del portafolio ha servido a los estudiantes para comprender mejor los conceptos fundamentales de la asignatura y su trascendencia en el contexto profesional; desarrollar con diferente grado de excelencia competencias genéricas como análisis, síntesis, creatividad, gestión del tiempo, expresión escrita y ampliación del lenguaje profesional. Asimismo ha potenciado la autodirección en el aprendizaje y la interacción con el profesor, contribuyendo a modificar su imagen:

"Gracias a este nuevo método de estudio que he 
recibido voy a aprovechar más en un futuro, las clases, tutorías y prácticas porque ahora veo al profesor como guía en mi aprendizaje”.

No obstante, la utilización del portafolio genera una serie de dificultades que pueden desmotivar al estudiante y ser causa de escepticismo, desinterés y abandono de la experiencia. Al igual que otros autores ${ }^{10,11}$ destacamos la inseguridad inicial provocada por la libertad de creación en una situación de aprendizaje tan abierta. Sin embargo, lo que al principio suponía una dificultad, se fue convirtiendo en ventaja ya que les permitió focalizar en aquellos conceptos que para cada uno resultaban más complicados.

Para los estudiantes, habituados a un aprendizaje receptivo, supuso también un obstáculo identificar recursos diferentes a los propuestos y gestionar su tiempo de estudio. Igualmente, todos coincidieron en que se incrementó su tiempo de trabajo frente al utilizado con métodos tradicionales, lo que resulta lógico ya que realizaron un aprendizaje profundo y no tenían experiencia como aprendices activos. No obstante, hay que tener en cuenta que esta asignatura tiene 7 créditos ECTS, que aproximadamente equivale a 175 horas de trabajo del estudiante, lo que se acerca mucho a los tiempos expresados por la mayoría.

Consideramos que el portafolios constituye un instrumento de evaluación formativa muy eficaz, adquiriendo protagonismo la autoevaluación y coevaluación continua del proceso de aprendizaje. A través del mismo, se obtuvo un material muy diversificado que reflejaba claramente la evolución del aprendizaje del estudiante, permitiendo identificar áreas de dificultad para su mejora progresiva a lo largo del curso, siendo "una ventana que se abre, no sólo ante el trabajo del estudiante, sino ante su manera de pensar"11.

La utilización de este instrumento requiere mayor tiempo de dedicación del profesor para cada estudiante que las clases presenciales, de forma que para llevarla a cabo con los 80-90 estudiantes matriculados en la asignatura, sería necesario contar con apoyos para revisar portafolios y realizar las tutorías. No obstante, pensamos que se puede hacer en pequeños grupos y que las tutorías planificadas deben ser conjuntas, tanto para potenciar el aprendizaje cooperativo como para optimizar el tiempo del profesor.

\section{BIBLIOGRAFÍA}

1. Klenowxki V. Desarrollo de portafolios para el aprendizaje y la evaluación. Madrid: Narcea; 2004.

2. Billings D, Kowalski K. Portafolioss learning.The Journal of Continuing Education in Nursing 2005; 36 (4): 149-150.

3. Alexander J, Craft S, Baldwin S, Beers G, McDaniel G. The nursing portfolio: A reflection of a professional .The Journal of Continuing Education in Nursing 2002; 33 (2): 55-60.

4. Olive M. Implementación del portafolio del estudiante en la asignatura "fonaments d'infermeria" como instrumento para el aprendizaje reflexivo y crítico. E.ducare 21 2004; 13: 1-15.

5. Font A.Una experiencia de autoevaluación y evaluación negociada en un contexto de aprendizaje basado en problemas. Revista de la REDU; 3 (2): 100-112

6. Klenowxki V. Desarrollo de portafolios para el aprendizaje y la evaluación. Madrid: Narcea; 2004. p.45.

7. Sorrell J, Brown H, Silva M, Kohlenberg E.Use of writing portfolios for interdisciplinary assessment of critical thinking outcomes of nursing students. Nursing Forum 1997; 32 (4): 12-25.

8. Williams J.The clinical notebook: Using student portfolios to enhance clinical teaching learning. Journal of Nursing Education 2001; 40(3): 135-138.

9. Schaffer M, Nelson P, Litt E.Using portfolios to evaluate achievement of population-based public health nursing competencies in baccalaureate nursing students. Nursing Education Perspectives 2005; 26 (2): 104-113.

10. Olive M, Gusiñe C, Pardo A. El portafolio como instrumento para favorecer el aprendizaje reflexivo, organización y opinión del estudiante. En http://www.upf.edu/bolonya/butlletins/ 2005/marc1/opinion.pdf. Fecha de acceso: Julio, 2006.

11. Mcmullan M. Students' perceptions on the use of portfolios in pre-registration nursing education: A questionnaire survey. International Journal of Nursing Studies 2006; 43 (3): 333343.

12. Agra MJ, Gewerc A, Montero L. El portafolios como herramienta de análisis en experiencias de formación on line y presenciales. En web.udg.es/tiec/orals/c45.pdf. Fecha de acceso: Julio, 2006. 